\title{
Depression diagnoses following the identification of bipolar disorder: costly incongruent diagnoses
}

\author{
Michael D Stensland ${ }^{1}$, Jennifer F Schultz ${ }^{2}$ and Jennifer R Frytak*3
}

\begin{abstract}
Background: Previous research has documented that the symptoms of bipolar disorder are often mistaken for unipolar depression prior to a patient's first bipolar diagnosis. The assumption has been that once a patient receives a bipolar diagnosis they will no longer be given a misdiagnosis of depression. The objectives of this study were 1) to assess the rate of subsequent unipolar depression diagnosis in individuals with a history of bipolar disorder and 2) to assess the increased cost associated with this potential misdiagnosis.

Methods: This study utilized a retrospective cohort design using administrative claims data from 2002 and 2003. Patient inclusion criteria for the study were 1) at least 2 bipolar diagnoses in 2002,2) continuous enrollment during 2002 and 2003, 3) a pharmacy benefit, and 4) age 18 to 64. Patients with at least 2 unipolar depression diagnoses in 2003 were categorized as having an incongruent diagnosis of unipolar depression. We used propensity scoring to control for selection bias. Utilization was evaluated using negative binomial models. We evaluated cost differences between patient cohorts using generalized linear models.

Results: Of the 7981 patients who met all inclusion criteria for the analysis, 17.5\% (1400) had an incongruent depression diagnosis (IDD). After controlling for background differences, individuals who received an IDD had higher rates of inpatient and outpatient psychiatric utilization and cost, on average, an additional \$1641 per year compared to individuals without an IDD.

Conclusions: A strikingly high proportion of bipolar patients are given the differential diagnosis of unipolar depression after being identified as having bipolar disorder. Individuals with an IDD had increased acute psychiatric care services, suggesting higher levels of relapses, and were at risk for inappropriate treatment, as antidepressant therapy without a concomitant mood-stabilizing medication is contraindicated in bipolar disorder. Further prospective research is needed to validate the findings from this retrospective administrative claims-based analysis.
\end{abstract}

\section{Background}

Bipolar disorder, a severe and recurrent mental disorder, is characterized by episodes of elated and depressed mood. Epidemiological studies have reported lifetime prevalence ranging from $0.8 \%-5.1 \%$ [1-3]. However, in most private claims databases, the prevalence of treated bipolar disorder has been found to be lower $(0.2 \%)[4,5]$. This discrepancy can be attributed to 2 factors: Only $40 \%$ of individuals with bipolar disorder have private insurance [6], and many patients are not correctly diagnosed.

The results of screening studies for bipolar disorder have shown that a strikingly high proportion of individuals seeking treatment for symptoms of bipolar disorder

\footnotetext{
* Correspondence: jennifer.frytak@i3innovus.com

3 i3 Innovus, Eden Prairie, Minnesota, USA

Full list of author information is available at the end of the article
}

are not diagnosed. In a recent primary care screening study, less than $10 \%$ of individuals who screened positive for bipolar disorder on a brief screening tool (Mood Disorders Questionnaire; MDQ) reported being previously diagnosed with bipolar disorder [7]. In another study that rigorously confirmed the bipolar diagnosis, $25.6 \%$ of psychiatric outpatients with bipolar I and $50.5 \%$ with bipolar II disorder were not diagnosed [8]. Other survey research found an average time lag between onset of symptoms and diagnosis of 7-10 years [6,8].

Part of the challenge of recognizing bipolar disorder is differentiating it from other disorders, particularly nonbipolar, or unipolar, depression [9], given the high degree of symptom overlap. The symptoms a bipolar patient experiences during a depressive episode meet the diagnostic criteria for major depressive disorder; the disor- 
ders are differentiated based on the patient's history of manic or hypomanic symptoms [10]. Unfortunately, patients often do not recall past manic symptoms or do not recall them as problematic [11]. Further, depressive symptoms are present 3 times as often as manic symptoms in patients with bipolar disorder [12]. Thus, eliciting a history of manic or hypomanic symptoms is a difficult challenge for clinicians. Yet, when such a history remains unknown, patients are likely to receive a unipolar depression diagnosis and treatment that is inappropriate or contraindicated for bipolar disorder, such as antidepressant monotherapy and lack of appropriate mood-stabilizing medication.

Because of the important treatment implications of this differential diagnosis, efforts have been made to improve initial identification of bipolar disorder and differentiate it from unipolar depression. Review articles have described the subtle clinical characteristics that differentiate not-yet-recognized bipolar disorder from unipolar depression [13,14]. In addition, screening tools for bipolar disorder, such as the MDQ [15] and a claims-based screening algorithm [16], have been developed to help identify unrecognized bipolar disorder.

These efforts assumed that an accurate diagnosis of bipolar disorder, once achieved, would remain with the patient throughout future treatment, but our previous research suggests that an initial diagnosis of bipolar disorder may be less stable than previously thought. We found that $27.5 \%$ of individuals initially diagnosed with bipolar disorder received unipolar depression disorder diagnoses after they had been diagnosed with bipolar disorder $[17,18]$. Those patients who had received incongruent depression diagnoses (IDDs) had an $82 \%$ increase in mental health hospitalizations, a $147 \%$ increase in mental health emergency room (ER) visits, and an $80 \%$ increase in mental health ambulatory visits, resulting in an increase of $\$ 3189$ per patient per year in treatment costs relative to those patients who were not given the incongruent unipolar depression diagnosis. Analysis of provider switching revealed that the lack of continuity of care among mental health providers was the most convincing mechanism for the loss of the bipolar diagnosis.

Our earlier study [18], selected a population of individuals who had been newly diagnosed with bipolar disorder in their administrative claims. However, a health management intervention study to validate those findings would be simpler to implement and potentially have larger cost savings if conducted in the larger population of all individuals with a diagnosis of bipolar disorder, rather than just those newly diagnosed. This potential intervention could start on a given date, examine all individuals with a history of a bipolar diagnosis, screen for new claims with depression diagnoses from a different healthcare provider, and then intervene to inform the provider of the previous bipolar disorder diagnosis. Identifying the best population to intervene in is of paramount concern for designing a health management intervention.

The objectives of the current study were to identify the costs of an incongruent diagnosis by expanding the study population from initially diagnosed bipolar patients to all bipolar patients. Specifically, we assessed the rate of IDDs given to individuals with a history of bipolar disorder as of January 1, 2003 and assessed the increased costs associated with the IDD. We intend to inform the design and population for a potential intervention by analyzing this study population with similar methods from our prior research.

\section{Methods}

This study design used retrospective, longitudinal claims data from a large, national, managed-care organization providing coverage for inpatient care, ambulatory services, and prescription drugs. The study sample was derived from commercially insured health plan members or members with Medicaid managed-care coverage, 18 to 64 years of age, who had medical and pharmacy benefits, and who were continuously enrolled in the health plan from January 1, 2002 until December 31, 2003. The data were used with permission from the data source. Individuals may not have had continuous enrollment during the study period for a variety of reasons including, but not limited to, a loss of employment, a switch in employers, an employer's switching of insurance companies, failure to pay insurance premiums, discontinuation of insurance coverage, or death. Study patients were required to have a minimum of 2 bipolar diagnoses in 2002. Because we used a prevalence-based sample rather than patients newly diagnosed, the index date was set to January 1 , 2003 for all patients. With the exception of the definition of the index date and the precise time period for continuous enrollment, the study methods and variable definitions mirror those of our previously published study [18].

To control for background differences between IDD and no incongruent depression diagnosis (NIDD) patients, predicted probabilities were used as a covariate in the outcome models [19]. The predicted probabilities were calculated from a backward elimination logistic regression predicting IDD status based on variables measured in the baseline period. Backward elimination was used to identify the covariates and to reduce the potential for bias from multicollinearity and endogeneity. The independent variables in the model were measured during the baseline period and are listed in Table 1.

Negative binomial regression models were used to investigate the differences in the number of mental health providers, general practitioners (GPs), and other providers in the follow-up period across the 2 cohorts, controlling for baseline covariates, including the number of 
Table 1: Descriptive statistics: demographic, utilization, and cost variables

\begin{tabular}{|c|c|c|c|c|c|c|}
\hline & & & & & & \\
\hline & NID & $6581)$ & IDD & 1400) & Uni- variate & Multivariate \\
\hline Demographic Variables: & & & & & $p$ & $\mathrm{p}$ \\
\hline Men, $n, \%$ & 2378 & $36.13 \%$ & 391 & $27.93 \%$ & $<.0001$ & \\
\hline Age, mean, SD & 40.62 & 11.02 & 39.73 & 10.97 & .0064 & .0317 \\
\hline Region & & & & & & \\
\hline Northeast, n, \% & 747 & $11.35 \%$ & 213 & $15.21 \%$ & $<.0001$ & \\
\hline South, $n, \%$ & 2781 & $42.26 \%$ & 570 & $40.71 \%$ & .2879 & .0845 \\
\hline West, n, \% & 860 & $13.07 \%$ & 159 & $11.36 \%$ & .0816 & \\
\hline Midwest, n, \% & 2193 & $33.32 \%$ & 458 & $32.71 \%$ & .6605 & \\
\hline Plan Type & & & & & & \\
\hline Commercial, n, \% & 6232 & $94.70 \%$ & 1344 & $96.00 \%$ & .0437 & \\
\hline Medicaid, n, \% & 349 & $5.30 \%$ & 56 & $4.00 \%$ & .0437 & \\
\hline Baseline Variables: & & & & & & \\
\hline Baseline Unipolar Dx, n, \% & 886 & $13.46 \%$ & 936 & $66.86 \%$ & $<.0001$ & $<.0001$ \\
\hline Number of Unipolar Dxs ${ }^{a}$ & 0.95 & 3.24 & 8.96 & 11.60 & $<.0001$ & $<.0001$ \\
\hline Total Costs ${ }^{\mathrm{a}}$ & 9056.62 & 18371.86 & 13448.57 & 20021.63 & $<.0001$ & .0163 \\
\hline M H Ambulatory Costa & 835.59 & 1704.91 & 1999.31 & 4421.06 & $<.0001$ & $<.0001$ \\
\hline Non-MH Ambulatory Costa ${ }^{a}$ & 2089.39 & 4000.50 & 2835.56 & 5239.78 & $<.0001$ & .0652 \\
\hline Mental Health ER Cost ${ }^{a}$ & 48.42 & 304.59 & 96.40 & 525.63 & .001 & \\
\hline Non-MH ER Costa & 206.93 & 845.65 & 293.98 & 1041.55 & .0034 & \\
\hline MH Inpatient Cost ${ }^{\mathrm{a}}$ & 793.66 & 3428.65 & 2141.36 & 7325.41 & $<.0001$ & \\
\hline Non-MH Inpatient Cost ${ }^{\mathrm{a}}$ & 1752.31 & 17979.46 & 1658.32 & 12441.45 & .8141 & \\
\hline MH Medication Costa & 1745.42 & 1909.65 & 2153.20 & 2032.10 & $<.0001$ & .018 \\
\hline Total Medication Costa & 2836.37 & 2882.69 & 3545.40 & 3370.89 & $<.0001$ & \\
\hline Number of Psychotherapy Sessions ${ }^{a}$ & 5.92 & 9.43 & 13.14 & 13.77 & $<.0001$ & \\
\hline Antidepressant Day Supplya & 186.58 & 196.25 & 276.30 & 209.07 & $<.0001$ & $<.0001$ \\
\hline Lithium Day Supplya & 55.36 & 111.00 & 31.05 & 81.58 & $<.0001$ & $<.0001$ \\
\hline Benzodiazepine Day Supplya & 64.38 & 123.73 & 98.61 & 144.33 & $<.0001$ & .0015 \\
\hline Anticonvulsants Day Supplya & 137.94 & 171.75 & 132.97 & 162.30 & .3035 & $<.0001$ \\
\hline Antidepressant Monotherapy ${ }^{b}$ & 0.082 & 0.27 & 0.10 & 0.30 & .031 & .0163 \\
\hline Number of Claims w/ADHD Dxa & 0.21 & 1.51 & 0.29 & 2.14 & .2126 & .0716 \\
\hline $\begin{array}{l}\text { Number of Claims with Non-MH Unique } \\
\text { Dx }\end{array}$ & 12.62 & 9.38 & 15.32 & 10.47 & $<.0001$ & .0403 \\
\hline Index BP Dx on Inpatient Claim, n, \% & 312 & $4.74 \%$ & 163 & $11.64 \%$ & $<.0001$ & $<.0001$ \\
\hline BP Diagnosis on ER Claim, $n, \%$ & 273 & $4.15 \%$ & 28 & $2.00 \%$ & .0001 & .0225 \\
\hline Index BP Dx Mixed Episode, n, \% & 1208 & $18.36 \%$ & 222 & $15.86 \%$ & .0269 & .0584 \\
\hline Index BP Dx Unspecified Episode, n, \% & 3020 & $45.89 \%$ & 574 & $41.00 \%$ & .0008 & .0094 \\
\hline Charlson Comorbidity Indexa & 0.51 & 1.14 & 0.58 & 1.19 & .042 & \\
\hline
\end{tabular}

aln the 1-year period prior to index bipolar disorder diagnosis, mean, SD.

bPatients treated with antidepressant monotherapy were coded 1 ; those who were not treated with antidepressant monotherapy were coded 0 , mean, SD.

$\mathrm{NIDD}=$ no incongruent depression diagnosis; IDD = incongruent depression diagnosis; $\mathrm{SD}=$ standard deviation; $\mathrm{Dx}=$ diagnosis; $\mathrm{MH}=\mathrm{mental}$ health; $\mathrm{ER}=$ emergency room; $\mathrm{ADHD}=$ attention deficit/hyperactivity disorder; $\mathrm{BP}=$ bipolar 
providers (mental health, GP, other) in the baseline period. Health care utilization was estimated using negative binomial models. Two-part models were used to analyze the relationship between IDD and health care costs. These models deal with the unique characteristics of medical expenditure data, which are typically skewed and censored. The first step was to estimate whether individuals had any medical expenditures using logistic regression. In the second step, a generalized linear model (GLM) was used to estimate positive costs. GLMs account for non-constant variance and maintain the original scale of the data, thus eliminating the need to transform cost data to achieve homoskedasticity and the need to retransform using a Duan smearing estimator for interpreting results [20]. The results of the 2-part model were combined to predict medical expenses for an individual by multiplying the prediction from each part of the model (the probability of positive expenses times the predicted medical expense from the GLM specification) [21].

To integrate the 2-part model, we first derived predicted cost estimates by running 2 prediction models: the first assuming the entire sample had an IDD and the second assuming the entire sample did not. We then calculated predicted probabilities of health care utilization. Predicted costs were combined with predicted probabilities of having any resource utilization (to account for individuals with 0 visits and 0 costs).

To specify the cost models, we used a variant of the Park test to determine the appropriate GLM distribution and link function [22]. The gamma distribution with a log-link function was used to estimate positive costs. We calculated robust standard errors using the HuberWhite-type correction for the variance-covariance matrix of the parameter estimates.

The administrative claims data were statistically deidentified and compliant with the provisions of the Health Insurance Portability and Accountability Act (HIPPA) of 1996 standards. Therefore, this study did not require Institutional Review Board review.

\section{Results}

A total of 7981 patients diagnosed with bipolar disorder met all inclusion criteria for the analysis (see Figure 1). Of these patients, $1400(17.5 \%)$ were classified as having an IDD in the follow-up period. Approximately $66.9 \%$ (936/ $1400)$ of patients with an IDD and $13.5 \%(886 / 6581)$ of patients with NIDD in the follow-up period had a unipolar depression diagnosis during the baseline period. Descriptive statistics and statistical analyses of means and proportions on select variables for the 2 cohorts are shown in Table 1. A backward elimination logistic regression using the baseline variables from Table 1 to predict the likelihood of receiving an IDD in the follow-up period was relatively accurate. The area under the ROC curve

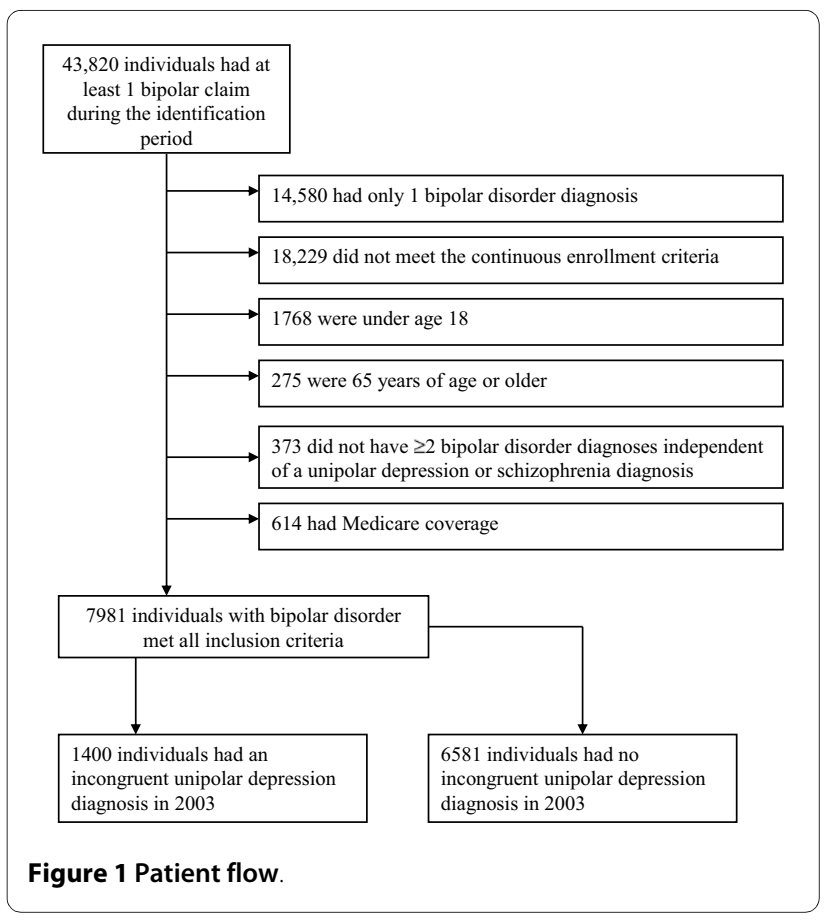

indicates that the background variables were able to accurately classify a randomly selected individual according to IDD status $84 \%$ of the time. Presence of a unipolar depression diagnosis in the baseline period was a particularly strong predictor (Odds Ratio $=4.6$ ) of a depression diagnosis in the follow-up period.

We analyzed the specialties of health care providers giving the first unipolar depression diagnosis (of the 2 required for our definition of incongruent diagnosis) to see if they differed from the specialties of health care providers who gave the first diagnosis of bipolar disorder in the baseline period. Within the IDD cohort, 1046 patients (74.1\%) received their bipolar disorder diagnosis from a mental health provider. Surprisingly, an even greater number of patients $(1144,81.7 \%)$ received the first of the 2 defined unipolar depression diagnoses from mental health providers; 93 patients $(6.6 \%)$ received this first unipolar diagnosis from GPs, and 163 (11.6\%) from other providers (hospitals, internal medicine, emergency medicine, or unknown). In 1070 cases (76.4\%), the physician giving the IDD had not previously diagnosed the patient with bipolar disorder.

The number of health care providers seen by patients in the follow-up period differed significantly between patient cohorts. IDD patients saw an average of 2.4 (standard deviation $[\mathrm{SD}]=1.7)$ mental health care providers versus $1.2(\mathrm{SD}=1.2)$ for NIDD patients $(\mathrm{p}=.001)$. After controlling for predicted probability and number of mental health practitioners in 2002, the number of visits with a mental health care provider was 1.83 times greater for IDD than for NIDD patients. Similar results were found 
for general practitioners (IDD: $1.4 \pm 1.6$; NIDD: $1.2 \pm 1.3$; $\mathrm{p}=.003$, Relative risk $[\mathrm{RR}]=1.14$ ) and for all other practitioners (IDD: $7.8 \pm 7.6$; NIDD: $5.7 \pm 5.9$; $\mathrm{p}=.001$, RR = 1.13).

IDD patients had significantly more ambulatory mental health visits, inpatient mental health visits, and ER mental health visits in the follow-up period compared to NIDD patients (see Table 2) after controlling for baseline covariates. The RRs from the models indicated that the average number of mental health ambulatory visits was 1.74 times higher for IDD patients than for NIDD patients. The mean number of mental health hospital visits and ER visits were 3.06 and 2.06 times greater, respectively, for the IDD patients. In addition, IDD patients' mental health ambulatory visits were $73 \%$ more expensive (see Table 3).

Figure 2 shows the cost differences for the various components based on this integration of the 2-part model. The largest cost difference between the 2 cohorts is for inpatient mental health care (\$1365 for IDD; \$608 for NIDD; difference of \$757). If all patients in the study received an incongruent diagnosis, average total treatment costs per person per year would be $\$ 10,773$. If patients did not have the IDDs, average total treatment costs would be $\$ 9132$. Thus, the treatment costs associated with an IDD were $\$ 1641$ per person per year.

\section{Discussion}

This study replicates our previous finding that a meaningful proportion of individuals with a bipolar diagnosis were given a subsequent incongruent unipolar depression diagnosis and had increased treatment costs. These results extend our previous finding from initially diagnosed to all bipolar patients. In 2003, 17.5\% (1400/7981) of individuals who had been previously diagnosed with bipolar disorder were diagnosed with unipolar depression, a differential diagnosis. Diagnostic criteria indicate that once a person exhibits symptoms of mania or hypomania that person has bipolar disorder; all future depressive symptoms are part of the bipolar disorder rather than unipolar depression [10]. The IDDs were associated with a $\$ 1641$ increase in treatment costs per patient, after correcting for background differences.

Given that patients were not randomized to be given a misdiagnosis of unipolar depression in a controlled study, we cannot be certain that the increased costs were due to the apparent misdiagnoses. For obvious practical and ethical reasons, one cannot complete a controlled study in which participants are randomized to be misdiagnosed. Although we corrected for background differences between the groups on January 1, 2003, we cannot be certain that the differences between groups in 2003 were due to the IDDs that occurred during 2003. The individuals with IDDs may have simply had more health care interactions in 2003 and therefore more opportunity for an incongruent diagnosis and increased costs. However, we find the misdiagnoses explanation more plausible for a variety of reasons.

The pattern of increased treatment costs is indicative of greater psychiatric relapses (see Figure 2). The 3-fold increase in rate of psychiatric hospitalization and 2-fold increase in psychiatric ER visits (see Table 2) strongly suggest that the individuals who receive the IDDs have more psychiatric relapses. The increased psychiatric outpatient and medication costs that would be expected for individuals experiencing relapses are also observed, although these costs are more difficult to interpret as they can increase for other reasons as well, such as individuals becoming more engaged in treatment.

Table 2: Average number of visits by incongruent depression diagnosis in 2003

\begin{tabular}{|c|c|c|c|c|}
\hline \multirow[b]{3}{*}{ Visit Type } & \multicolumn{2}{|c|}{ Cohort } & \multirow[b]{3}{*}{$\mathbf{R R}^{\mathbf{a}}$} & \multirow[b]{3}{*}{$\mathbf{p}$} \\
\hline & NIDD & IDD & & \\
\hline & Predicted Mean & Predicted Mean & & \\
\hline MH Ambulatory & 6.57 & 11.46 & 1.74 & $<.0001$ \\
\hline Non-MH Ambulatory & 10.08 & 10.56 & 1.05 & .22 \\
\hline MH Hospital & 0.27 & 0.56 & 3.06 & $<.0001$ \\
\hline Non-MH Hospital & 0.11 & 0.12 & 1.16 & .26 \\
\hline MHER & 0.06 & 0.19 & 2.06 & $<.0001$ \\
\hline Non-MH ER & 0.61 & 0.73 & 1.20 & .17 \\
\hline
\end{tabular}

aRelative risk of increased visits for IDD cohort relative to NIDD cohort after correcting for background differences. $\mathrm{NIDD}=$ no incongruent depression diagnosis; IDD = incongruent depression diagnosis; $\mathrm{RR}=$ relative risk; $\mathrm{MH}=\mathrm{mental}$ health; $\mathrm{ER}=$ emergency room 
Table 3: Cost per patient by cohort for individuals who used the resource type

\begin{tabular}{|c|c|c|c|c|c|c|}
\hline \multirow[b]{3}{*}{ Visit Type } & \multicolumn{2}{|c|}{ Cohort } & & & \multicolumn{2}{|c|}{ Cohort } \\
\hline & IDD & NIDD & & & IDD & NIDD \\
\hline & $\begin{array}{r}\text { Predicted Mean } \\
(\text { costs }>0)\end{array}$ & $\begin{array}{r}\text { Predicted Mean } \\
(\text { costs }>0)\end{array}$ & $\mathbf{R R}^{\mathbf{a}}$ & $\mathbf{p}$ & $\begin{array}{l}\text { Probability of } \\
\text { Resource Use }\end{array}$ & $\begin{array}{l}\text { Probability of } \\
\text { Resource Use }\end{array}$ \\
\hline Total Cost $(\mathrm{N}=7900)$ & $\$ 10,773$ & $\$ 9132$ & 1.18 & .005 & & \\
\hline MH Ambulatory $(\mathrm{N}=6249)$ & 1422 & 821 & 1.73 & $<.0001$ & 0.96 & 0.74 \\
\hline Non-MH Ambulatory ( $\mathrm{N}=7332)$ & 2462 & 2664 & 0.92 & .34 & 0.94 & 0.91 \\
\hline MH Hospital $(\mathrm{N}=589)$ & 9491 & 7837 & 1.00 & .97 & 0.18 & 0.05 \\
\hline Non-MH Hospital ( $\mathrm{N}=675)$ & 15,704 & 14,841 & 1.06 & .77 & 0.10 & 0.08 \\
\hline MH Emergency Room ( $\mathrm{N}=644)$ & 658 & 644 & 1.02 & .88 & 0.12 & 0.07 \\
\hline Non-MH Emergency Room ( $\mathrm{N}=1509)$ & 1100 & 1045 & 1.05 & .68 & 0.23 & 0.18 \\
\hline Mental Health Prescription $(\mathrm{N}=7014)$ & 2272 & 2299 & 0.99 & .75 & 0.95 & 0.86 \\
\hline Total Prescription $(\mathrm{N}=7679)$ & 3522 & 3454 & 1.02 & .59 & 0.98 & 0.96 \\
\hline
\end{tabular}

aRelative risk of increased costs for IDD cohort relative to NIDD cohort after correcting for background differences. Note: Generalized Linear Models with Log Link Specification

$\mathrm{NIDD}=$ no incongruent depression diagnoses; IDD = incongruent depression diagnoses; $\mathrm{MH}=$ mental health

When a patient with bipolar disorder is misdiagnosed with unipolar depression, the resulting treatment will likely be contraindicated. The primary pharmacologic treatment for unipolar depression is antidepressant monotherapy. Well-controlled clinical trials have found that antidepressant monotherapy, particularly with tricyclic antidepressants, in patients with bipolar disorder can induce mania at a higher rate than placebo [23]. Further, unipolar depression is generally not treated with moodstabilizing medications, which represent the hallmark of treatment for bipolar disorder. Thus, appropriate pharmacologic treatment and control of symptoms depend on an accurate diagnosis of the patient's bipolar disorder.

Analysis of the number of providers and provider switching supports the notion that IDDs result, in part,

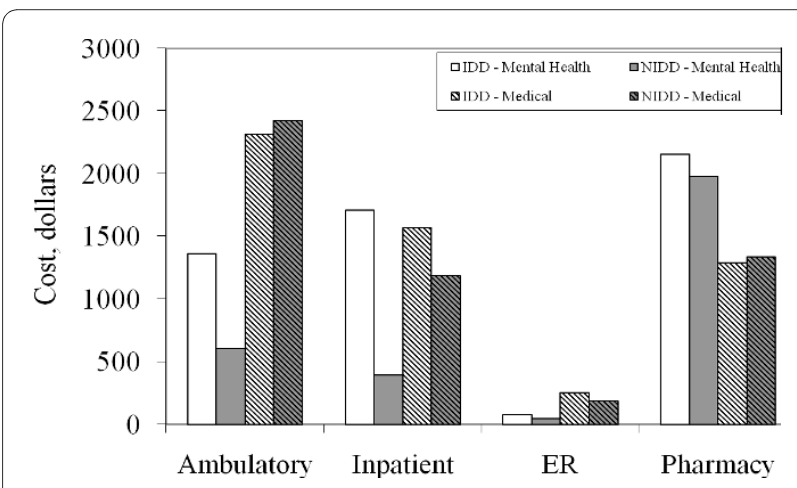

Figure 2 Differences in cost components for individuals with incongruent depression diagnosis from continuity of care issues as patients with this episodic and diagnostically challenging disorder interact with the health care system. Individuals receiving IDDs had twice as many mental health providers as those who did not receive IDDs. Furthermore, in $76 \%$ of the cases, the provider who gave the first incongruent unipolar depression diagnosis had not previously given the patient a bipolar diagnosis. Continuity of care may be especially important for patients with bipolar disorder, because they often do not recall manic symptoms or do not recall them as problematic [11]. Given that less than half of patients discharged after medical hospitalization are able to correctly state their diagnosis [24] and that medical records are often not received when requested [25], the physician giving the IDD may not have information concerning the patient's previous manic or hypomanic symptoms, especially when the patient is new to the physician's practice.

Interestingly, the providers making the IDD were generally mental health specialists (psychiatrists [47.7\%], psychologists [12.8\%], social workers [20.5\%], other mental health personnel [0.71\%]). We would expect that most of these individuals are well educated about the symptoms and presentation of bipolar disorder, which suggests that this rate of IDDs results from the daunting task of differentiating the 2 disorders at a given point in time rather than a lack of knowledge about bipolar disorder. In the absence of information about past manic symptoms, a diagnosis of the more prevalent unipolar depression is more reasonable.

Given that mental health providers made the majority of IDDs, educational efforts to increase the awareness of the symptoms and presentation of bipolar disorder would 
probably only minimally reduce the rate of IDDs. On the other hand, education about the high rates of IDDs and the risk factors associated with them may be more effective in this provider population. To be effective, an intervention needs to result in the current physician receiving and incorporating information about the past bipolar diagnosis or symptoms into the current diagnosis.

\section{Limitations}

This research utilized administrative claims data, which enabled the unobtrusive observation of usual clinical practice for a large number of individuals, a necessary condition for this research. However, administrative claims data have limitations given that the data was collected for reimbursement rather than research purposes. As a result, the measures in the data are not ideal, and the study design is limited to statistical rather than experimental control, leaving the data open to alternative explanations.

The diagnostic information in claims data may not be reliable. For some conditions, such as Alzheimer's disease [26] and myocardial infarction [27], claims diagnosis algorithms have been found to have high agreement with medical charts; however, the predictive value of our algorithm from claims diagnoses for bipolar disorder has not been demonstrated. Unützer and colleagues [5] conducted a chart review of individuals identified as having bipolar disorder based on various criteria in administrative claims and reported using an unspecified standard that a "reasonable" number of individuals with at least 1 inpatient discharge diagnosis or outpatient diagnosis had evidence of bipolar disorder in his or her medical chart. Our algorithm, which was more restrictive than the simpler criteria studied by Unützer and colleagues, required at least 2 diagnoses from hospital or physician visits that did not have exclusionary diagnoses and, therefore, should be at least "reasonably" accurate. However, even if the diagnoses in the claims match those in the patients' charts, they still may not coincide with the diagnoses made based on the gold standard Structured Clinical Interview for DSM-IV (SCID). Nonetheless, we believe our study population is representative, and our results can be generalized to similar populations.

Throughout this article, we have referred to the depression diagnoses following bipolar diagnoses as incongruent diagnoses rather than misdiagnoses. This has been in recognition that the diagnoses given in the claims may not reflect the true gold standard SCID diagnoses. In a recent study examining SCID diagnoses in outpatients, Zimmerman and colleagues found that less than half (43.4\%) of individuals reporting having been diagnosed with bipolar disorder met the SCID criteria for the disorder [28]. Interestingly, $30 \%$ of those that did meet the SCID diagnosis had not previously been diagnosed with bipolar disorder. These findings suggest that not only is bipolar often under-diagnosed it is also over-diagnosed. This raises the possibility that the IDD in our study may have been the correct diagnosis. However, given the pattern of resource utilization, we believe that the bipolar disorder diagnosis was more likely to correct on average. Previous research in private payer claims has found that bipolar disorder is more costly than unipolar depression, particularly in terms of psychotropic medication and psychiatric hospitalization costs [29]. If the unipolar depression diagnoses had been correct more often than the bipolar, we would have anticipated the IDD group to have lower, instead of higher, resource use, particularly for psychiatric hospitalization.

One potential alternative explanation for our results is individuals who received the IDD following a bipolar diagnosis were simply more complex patients who, to no surprise, incurred higher costs. In the analysis, we utilized predicted probabilities to statistically control for background differences between the IDD and NIDD patients. A large number of baseline variables were used to construct the predicted probabilities (Table 1). From a theoretical perspective, because these variables were used to calculate the predicted probabilities and the analysis adjusted for predicted probabilities, the difference between the IDD and NIDD could not have been driven by these background differences [19]. To the extent that these background variables, including costs, comorbidities, and resource use, capture patient "complexity", we have ruled out this as a driver of the result. However, if another confounding variable exists that was not included in the predicted probability calculation, it could still explain our results. A study of an intervention in which administrative claims are screened and physicians are contacted when they file a claim with an IDD is needed to validate our results and accurately assess the cost savings that could be realized.

\section{Conclusions}

An incongruent diagnosis of unipolar depression in persons previously identified with bipolar disorder appears to be relatively frequent and costly. Patients who received IDDs had increased psychiatric hospitalizations, ER visits, and ambulatory services. The apparent misdiagnosis may have resulted in patients not receiving the needed mood-stabilizing medications or receiving contraindicated antidepressant monotherapy. In this study, the IDDs appeared to arise when patients with bipolar disorder switch mental health providers, and the new provider may not be receiving information about past manic/ hypomanic episodes needed to differentiate bipolar disorder from unipolar depression. This retrospective claims-based analysis needs to be validated with a prospective health management intervention study where an intervention occurs when an IDD is given to an individual who was historically diagnosed with bipolar disorder by a different provider. An effective intervention that informs a physician who submits a claim with a depression diag- 
nosis for a patient about the patient's previous treatment for bipolar disorder could potentially improve patient care and save, on average, \$1641 per patient per year in a managed-care population.

\section{Competing interests}

Funding for this project was provided by Eli Lilly and Company (Indianapolis, Indiana, USA), including the article-processing charge. Michael Stensland was a full-time employee of Eli Lilly and Company and a minor stockholder while developing this research. Jennifer Schultz received funding from i3 Innovus to conduct the data analysis and serves as a consultant for the organization. Jennifer Frytak is an employee of i3 Innovus.

\section{Authors' contributions}

MDS was involved with designing the study; interpreting the data; and manuscript writing and reviewing. JSS had full access to all the data in the study, completed the data analysis, and was involved with writing and reviewing of the manuscript. JRF was involved with designing the study, study implementation, interpreting the data, and reviewing the manuscript. All authors have read and approved the final manuscript.

\section{Acknowledgements}

This study was funded by Eli Lilly and Company.

\section{Author Details}

'Agile Outcomes Research, Inc, Rochester, Minnesota, USA, 2University of Minnesota, Department of Economics, Labovitz School of Business and Economics, Duluth, Minnesota, USA and 3i3 Innovus, Eden Prairie, Minnesota, USA

Received: 15 August 2008 Accepted: 4 June 2010

Published: 4 June 2010

\section{References}

1. Kessler RC, Berglund P, Demler O, Jin R, Merikangas KR, Walters EE: Lifetime prevalence and age-of-onset distributions of DSM-IV disorders in the National Comorbidity Survey Replication. Arch Gen Psychiatry 2005, 62:593-602.

2. Kessler RC, McGonagle KA, Zhao S, Nelson CB, Hughes M, Eshleman S, Wittchen HU, Kendler KS: Lifetime and 12-month prevalence of DSM-IIIR psychiatric disorders in the United States. Results from the National Comorbidity Survey. Arch Gen Psychiatry 1994, 51:8-19.

3. Weissman MM, Bland RC, Canino GJ, Faravelli C, Greenwald S, Hwu HG, Joyce PR, Karam EG, Lee CK, Lellouch J, Lépine JP, Newman SC, RubioStipec M, Wells JE, Wickramaratne PJ, Wittchen H, Yeh EK: Cross-national epidemiology of major depression and bipolar disorder. JAMA 1996, 276:293-299.

4. Peele PB, Xu Y, Kupfer DJ: Insurance expenditures on bipolar disorder: clinical and parity implications. Am J Psychiatry 2003, 160:1286-1290

5. Unutzer J, Simon G, Pabiniak C, Bond K, Katon W: The treated prevalence of bipolar disorder in a large staff-model HMO. Psychiatr Serv 1998, 49:1072-1078

6. Suppes T, Leverich GS, Keck PE, Nolen WA, Denicoff KD, Altshuler LL McElroy SL, Rush AJ, Kupka R, Frye MA, Bickel M, Post RM: The Stanley Foundation Bipolar Treatment Outcome Network. II. Demographics and illness characteristics of the first 261 patients. J Affect Disord 2001 67:45-59.

7. Das AK, Olfson M, Gameroff MJ, Pilowsky DJ, Blanco C, Feder A, Gross R, Neria Y, Lantiqua R, Shea S, Weissman MM: Screening for bipolar disorder in a primary care practice. JAMA 2005, 293:956-963.

8. Mantere O, Suominen K, Leppämäki S, Valtonen H, Arvilommi P, Isometsä E: The clinical characteristics of DSM-IV bipolar I and II disorders: baseline findings from the Jorvi Bipolar Study (JoBS). Bipolar Disord 2004, 6:395-405.

9. Hirschfeld RM, Lewis L, Vornik LA: Perceptions and impact of bipolar disorder: how far have we really come? Results of the national depressive and manic-depressive association 2000 survey of individuals with bipolar disorder. J Clin Psychiatry 2003, 64:161-174.

10. American Psychiatric Association: Diagnostic and Statistical Manual of Mental Disorders Fourth edition. Washington, DC: American Psychiatric Association; 2000. Text Revision
11. Hirschfeld RM: Bipolar depression: the real challenge. Eur Neuropsychopharmacol 2004, 14(Suppl 2):S83-S88.

12. Judd LL, Akiskal HS, Schettler PJ, Endicott J, Maser J, Solomon DA, Leon AC, Rice JA, Keller MB: The long-term natural history of the weekly symptomatic status of bipolar I disorder. Arch Gen Psychiatry 2002, 59:530-537.

13. Bowden $\mathrm{CL}$ : A different depression: clinical distinctions between bipolar and unipolar depression. J Affect Disord 2005, 84:117-125.

14. Swann AC, Geller B, Post RM, Altshuler L, Chang KD, Delbello MP, Reist C, Juster IA: Practical Clues to Early Recognition of Bipolar Disorder: A Primary Care Approach. Prim Care Companion J Clin Psychiatry 2005, 7:15-21.

15. Hirschfeld RM, Williams JB, Spitzer RL, Calabrese JR, Flynn L, Keck PE Jr, Lewis L, McElroy SL, Post RM, Rapport DJ, Russell JM, Sachs GS, Zajecka J: Development and validation of a screening instrument for bipolar spectrum disorder: the Mood Disorder Questionnaire. Am J Psychiatry 2000, 157:1873-1875.

16. Juster IA, Stensland M, Brauer L, Thuras P: Use of administrative data to identify health plan members with unrecognized bipolar disorder: a retrospective cohort study. Am J Manag Care 2005, 11:578-584.

17. Stensland MD, Schultz JS, Frytak JR: Depression diagnoses following bipolar diagnoses in claims data: incongruent diagnoses related to increased treatment costs. San Diego, CA; 2005. Paper presented at: 57th Institute on Psychiatric Services

18. Schultz JS, Stensland MD, Frytak JR: Diagnoses of unipolar depression following initial identification of bipolar disorder: a common and costly misdiagnosis. J Clin Psychiatry 2008, 69:749-758.

19. D'Agostino RB Jr: Propensity score methods for bias reduction in the comparison of a treatment to a non-randomized control group. Stat Med 1998, 17:2265-2281

20. Duan N: Smearing estimate: a nonparametric retransformation method. J Am Stat Assoc 1983, 78:605-610.

21. Blough DK, Madden CW, Hornbrook MC: Modeling risk using generalized linear models. J Health Econ 1999, 18:153-171.

22. Manning WG, Mullahy J: Estimating log models: to transform or not to transform? J Health Econ 2001, 20:461-494.

23. Prien RF, Klett CJ, Caffey EM Jr: Lithium carbonate and imipramine in prevention of affective episodes. A comparison in recurrent affective illness. Arch Gen Psychiatry 1973, 29:420-425.

24. Makaryus AN, Friedman EA: Patients' understanding of their treatment plans and diagnosis at discharge. Mayo Clin Proc 2005, 80:991-994.

25. Mitchell JE, Pyle RL, Hatsukami D: Requesting previous psychiatric records. Do they come and are they worth obtaining? J Nerv Ment Dis 1981, 169:364-366.

26. Taylor DH Jr, Fillenbaum GG, Ezell ME: The accuracy of Medicare claims data in identifying Alzheimer's disease. J Clin Epidemio/ 2002, 55:929-937.

27. Kiyota Y, Schneeweiss S, Glynn RJ, Cannuscio CC, Avorn J, Solomon DH: Accuracy of Medicare claims-based diagnosis of acute myocardial infarction: estimating positive predictive value on the basis of review of hospital records. Am Heart J 2004, 148:99-104

28. Zimmerman M, Ruggero CJ, Chelminski I, Young D: Is bipolar disorder overdiagnosed? J Clin Psychiatry 2008, 69:935-940.

29. Stensland MD, Jacobson JG, Nyhuis A: Service utilization and associated direct costs for bipolar disorder in 2004: an analysis in managed care. $J$ Affect Disord 2007, 101:187-193.

Pre-publication history

The pre-publication history for this paper can be accessed here: http://www.biomedcentral.com/1471-244X/10/39/prepub

doi: $10.1186 / 1471-244 X-10-39$

Cite this article as: Stensland et al., Depression diagnoses following the identification of bipolar disorder: costly incongruent diagnoses BMC Psychiatry 2010, 10:39 